\title{
Testicular sperm aspiration in men with presumed non-obstructive azoospermia: Analysis of FSH level as a predictor of successful sperm retrieval
}

\section{Original Article}

\author{
Alexander K Chow, Laurence A Levine \\ Department of Urology, Rush University Medical Center, Chicago
}

\begin{abstract}
Aim of Study: To evaluate the sperm-retrieval rate (SRR) of testicular sperm aspiration (TESA) for men with presumed non-obstructive azoospermia (NOA) without prior genetic testing or testicular biopsies and to examine the correlation of successful sperm retrieval and FSH level.

Patients and Methods: The complete records of 148 azoospermic men without clinical evidence of obstructive etiologies who underwent TESA from 1999 - 2016 were reviewed. Preoperative diagnostic biopsies were not obtained and thus men with presumed NOA were defined as having no evidence of obstruction by history or physical examination. Preoperative FSH and testosterone were obtained for all patients. Two subgroups of NOA were evaluated (Group 1 with FSH $>10$ IU/L and Group 2 with FSH $\leq 10$ ).

Results: Of our 148 patients, we identified 66 (45\%) in Group 1 and $82(55 \%)$ in Group 2. Sperm was successfully obtained from 19/66 (28.7\%) in Group 1 and 56/82 (68\%) in Group 2. Average FSH for Group 1 and Group 2 were 20.4 (10 -66) IU/L and $4.6(1.1-9.4) \mathrm{IU} / \mathrm{L}$ respectively. There were no postoperative adverse events including prolonged pain, infection, hematoma, hydrocele or testicular atrophy.

Conclusion: TESA appears to offer a reasonably high likelihood of viable sperm retrieval in men with NOA. Given the higher level of SRR for men with presumed NOA with normal range of FSH $(\leq 10$ IU/L), we recommend that TESA should be considered the initial approach to retrieve sperm in this population as it is a quicker, easier, less invasive and likely a less costly approach to micro-TESE.
\end{abstract}

Key Words: Azoospermia, FSH, ICSI, micro-TESE, sperm retrieval, TESA, testicular sperm aspiration

Received: 2 October 2017, Accepted: 5 November 2017

Corresponding Author: Alexander K Chow, MD, Department of Urology, Rush University Medical Center, Chicago Tel.: 312-942-4006, E-mail: Alexander_k_chow@rush.edu,drlevine@hotmail.com

ISSN: 2090-7265

\section{INTRODUCTION}

Azoospermia is defined as the absence of spermatozoa in the ejaculate following centrifuge analysis on at least two occasions. This phenomenon is observed in $1.9 \%$ of the general population and in $10-15 \%$ of infertile men $^{[1]}$. In obstructive azoospermia (OA), spermatogenesis is present in testicular tissue; hence, surgical extraction and aspiration represent effective measures to obtain sperm. However, the greater challenge comes in finding and extracting sperm from men with non-obstructive azoospermia (NOA). Although there is an absence of spermatozoa in the epididymis, vas deferens and ejaculate, salvageable sperm may remain within the testicular parenchyma and can be found in up to $50 \%$ of testes ${ }^{[2]}$.

Microscopic testicular sperm extraction (micro-TESE) has emerged as the favored approach for testicular sperm retrieval in men with NOA. Microscopic visual aid enables the surgeon to identify qualitative differences between the seminiferous tubules and areas of "good quality" are then excised and sent for identification of spermatozoa.

An alternative to open testicular biopsy is testicular sperm aspiration (TESA), which was initially used as a diagnostic tool in the evaluation of azoospermia. If performed properly, TESA can be used to sample areas of spermatogenesis that may be missed with a simple open biopsy (TESE). The technique enables the surgeon to reach broad areas within the testicle ${ }^{[3]}$. TESA can be done with testicular mapping in which the testicle is divided into a grid and aspiration is taken through each grid with separate punctures in even distributions ${ }^{[4]}$. Alternatively, TESA is done at our institution through a single puncture wound in which the trajectory of the aspiration needle is reoriented to diffusely sample in the upper, mid and lower aspect of the 
testicle. Typically, three to four aspirations are performed for each testicle.

In this study, we evaluate the efficacy and safety of our TESA experience as a therapeutic choice for the harvest and cryopreservation of sperm for intracytoplasmic sperm injection (ICSI) specifically for patients with presumed non-obstructive azoospermia based on clinical history and physical examination as genetic testing and diagnostic biopsy were not obtained.

\section{PATIENTS AND METHODS}

Prior to the study, appropriate paperwork was submitted and approved by the Rush University Medical Center Institutional Review Board. We performed a retrospective chart review of 219 men who underwent TESA and who had complete charts for review at a single tertiary care fertility clinic during the period of 1999 to 2016. Patients were selected for surgery if there was an absence of spermatozoa in their ejaculate on two centrifuged semen analyses evaluated by a certified andrologist. Routine preoperative diagnostic biopsy was not obtained as part of our sperm retrieval protocol. Microdeletion of the $\mathrm{Y}$ chromosome was not routinely obtained due to cost and inconsistent insurance coverage. All patients underwent physical examination of the genitalia and a hormonal profile assessment including a testosterone and FSH level prior to surgery. All FSH levels represented solely endogenous production as we excluded men with previous FSH supplementation. Physical examination included a thorough palpation of the spermatic cord to evaluate for the presence of a vas deferens or a clinically present varicocele. Testicular size was determined by measuring the longitudinal dimension of each testicle with a ruler. Schoor et al found that a longitudinal testicular length of greater than $4.6 \mathrm{~cm}$ has a $72 \%$ sensitivity and $78 \%$ specificity of diagnosing $\mathrm{OA}^{[5]}$. We used a similar criterion to exclude patients from the study if they had a documented longitudinal length of greater than $4.6 \mathrm{~cm}$ which would suggest $\mathrm{OA}$.

Seventy-one patients had evidence of obstructive azoospermia and were excluded from this study. The remaining 148 patients were defined as men with presumed NOA as those men had no clinical indications of obstruction by history or physical examination (i.e. vasectomy, evidence of epididymal fullness, congenital absence of the vas deferens, history of genital trauma, prior scrotal or epididymal surgery, testicular size $>4.6 \mathrm{~cm}$ ).

We broke down our presumed NOA population into two groups to examine the difference in spermatozoa retrieval rate (SRR) in those patients with normal range FSH compared to those with elevated FSH. The normal FSH reference range at our institution was $\leq 10 \mathrm{IU} / \mathrm{L}$. Those with classic features of NOA with an FSH $>10$ IU/L compose Group 1 whereas those with FSH $\leq 10 \mathrm{IU} / \mathrm{L}$ composed Group 2.
TESA success was defined as the presence of at least one mature, viable and motile testicular sperm per high power field (HPF). Post-extraction analysis was completed at one fertility center. Statistical analysis was done using Independent T-Test on SPSS version 22.

All TESA procedures were performed by the same surgeon using a previously described technique. ${ }^{4}$ Aspirations were performed with the patient under IV sedation with a local spermatic cord block. Prior to the procedures, patients received a single IV dose of a broad-spectrum antibiotic. The scrotum was then shaved and cleansed with betadine. The testicle was immobilized by the surgeon (Fig. 1).

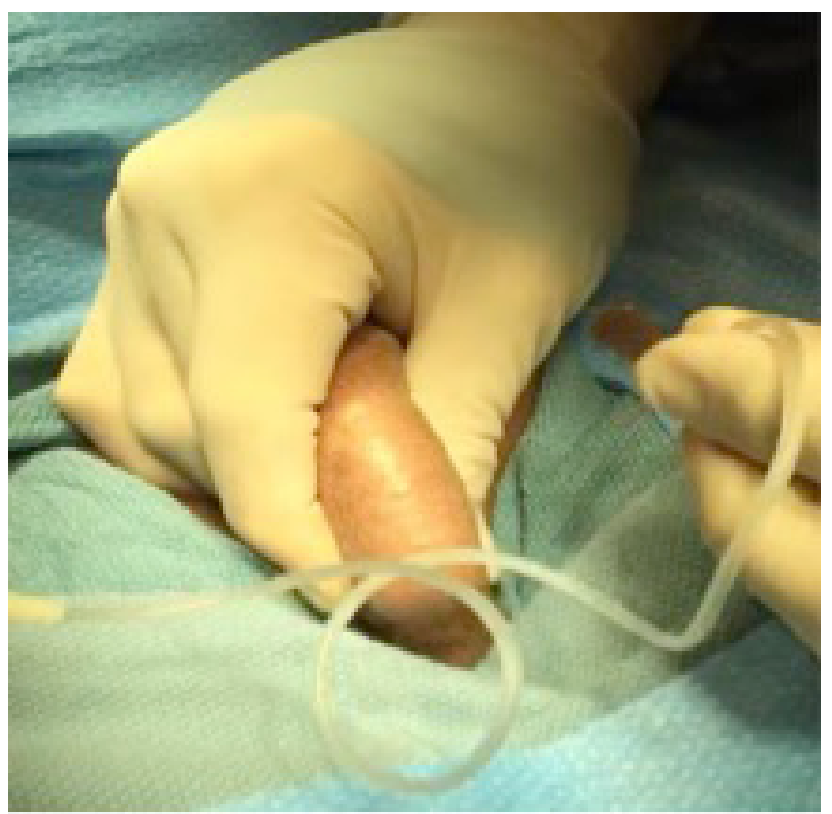

Sperm collection was performed with a 19-gauge butterfly needle attached to 12 -inch tubing. The needle was passed into the anterior-superior-lateral aspect of the testicle (Fig. 2).

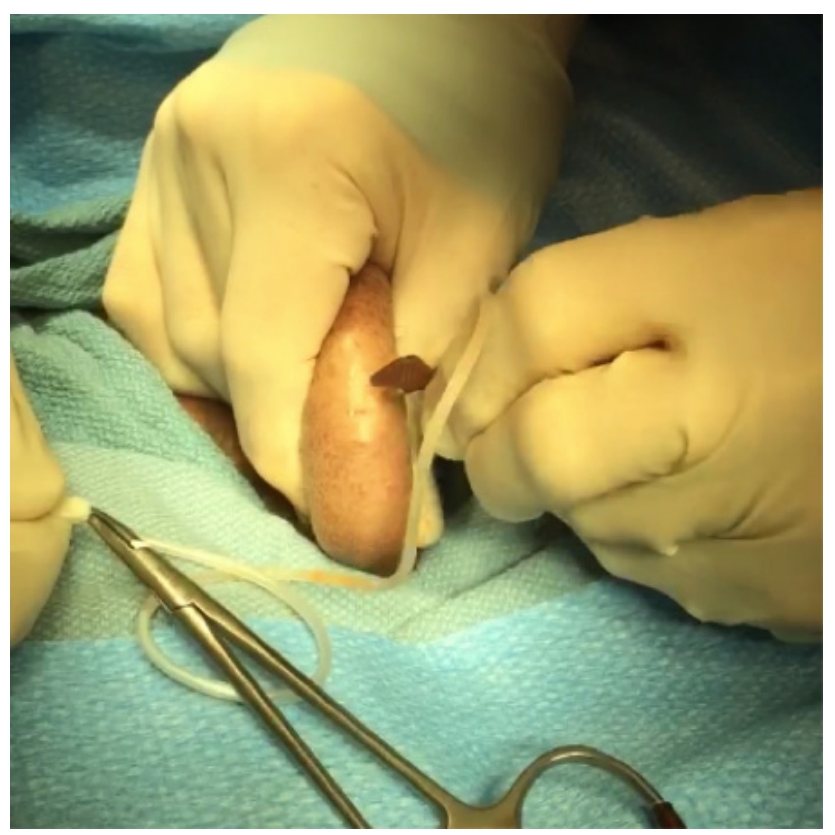


Suction was applied by pulling back on the plunger of a $30 \mathrm{cc}$ luer lock syringe. The tubing was then clamped to maintain suction. The needle was quickly advanced and pulled back in a vigorous thrusting fashion multiple times until opaque to yellow fluid and tissue was noted in the butterfly tubing. When a satisfactory specimen was obtained, the needle was removed and any tissue that remained attached to the needle tip was transected and included in the specimen which was placed in a sterile test tube with IVF media. This procedure was performed through the same skin puncture and the trajectory of the needle was slightly modified to sample the upper, middle

TESA Illustration and lower regions of the testicle. Typically, three to four aspirations were obtained per testicle, enough to diffusely sample the testicle. The specimen was then collected and sent for assessment in a single vial and thus mapping was not performed.

An immediate evaluation was performed by the in vitro fertilization team and used for sperm cryopreservation. In this study, success was defined as the presence of at least one viable and motile sperm per high power field after a freeze thaw cycle.
Sagittal cross section

A. Superior Pole Aspiration

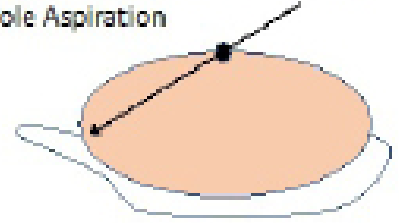

C. Mid Pole Aspiration

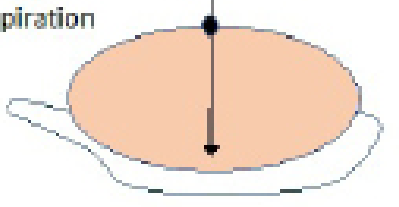

D. Inferior Pole Aspiration

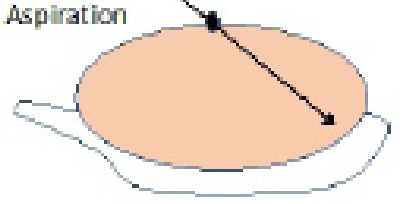

- Single puncture site

\section{Transverse cross section}

B.
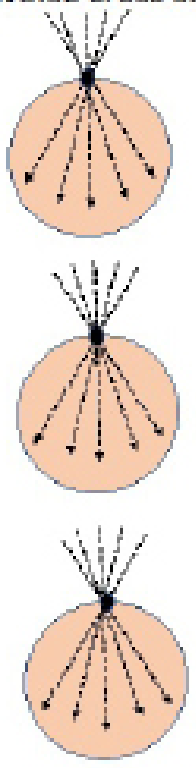

\section{LEGEND}

A. A 19 -gouge butterfly needle is passed through the anteriorsuperior-lateral aspect of the testicle.

B. As inclicated by the dotted lines, the needle is advanced and pulled back multiple times while changing trajectory trans versely to diffusely sample the testicle

C. Aspiration is repeated through the same puncture site to sample the mid pole.

D. Aspiration is repeated through the same puncture site to sample the inferior pole. 


\section{RESULTS}

Testicular sperm aspiration was performed on 148 men with presumed NOA as previously defined; 66 $(45 \%)$ in Group $1(\mathrm{FSH}>10 \mathrm{IU} / \mathrm{L})$ and $82(55 \%)$ in Group $2(\mathrm{FSH} \leq 10 \mathrm{IU} / \mathrm{L})$. The average age of Group 1 and Group 2 respectively was 33 (24-48) years and 35 (26-64) years. Group 1 had an average FSH of 20.4 (10 -66) whereas Group 2 had an average FSH of $4.6 \mathrm{IU} / \mathrm{L}(1.1-9.4)(\mathrm{p}=$ value 0.0001$)$. The average testosterone for Group 1 was 347 (23-864) compared to the average testosterone of Group 2 of 370 $(130-722)(p$ value $=0.751)$. Group 1 had an average testicular longitudinal length of $3.2 \mathrm{~cm}(2-4)$ whereas Group 2 had an average testicular longitudinal length of $3.4 \mathrm{~cm}(1.1-4.4)(\mathrm{p}=0.83)$. Identifiable pathology likely contributing to infertility included clinically palpable varicocele, primary testicular failure (documented prior to referral), testicular cancer status-post orchiectomy with adjuvant chemotherapy and/or radiation, cryptorchidism, testicular torsion status-post orchiectomy, mumps orchitis, Klinefelter syndrome, and Kallman syndrome (documented by karyotyping at another institution prior to referral). One patient with Kallman syndrome was treated with human chorionic gonadotropin to stimulate testosterone production. One patient with Klinefelter syndrome had FSH $<10$; this individual was receiving testosterone supplementation at the time of initial consultation. Testosterone supplementation was discontinued four months prior to undergoing TESA. See Table 1 for distribution of SRR for each pathology.

Sperm Retrieval Rate of Patients With Identifiable Pathology

\begin{tabular}{ccc}
\hline Pathology & & SRR \\
& & \\
\hline & Overall & $17 / 26(65 \%)$ \\
Varicocele & Group 1 & $11 / 17(65 \%)$ \\
& Group 2 & $6 / 9(67 \%)$ \\
Testicular CA s/p Orchiectomy +/- & Overall & $8 / 12(67 \%)$ \\
adjuvant chemotherapy and/or radiation & Group 1 & $5 / 5(100 \%)$ \\
& Group 2 & $3 / 7(43 \%)$ \\
Primary Testicular Failure & Overall & $4 / 11(36 \%)$ \\
& Group 1 & $1 / 7(14 \%)$ \\
Cryptorchidism & Group 2 & $3 / 4(75 \%)$ \\
& Overall & $4 / 7(57 \%)$ \\
& Group 1 & $2 / 5(40 \%)$ \\
Testicular Torsion Status-Post Orchiectomy & Group 2 & $2 / 2(100 \%)$ \\
& Overall & $1 / 2(50 \%)$ \\
Mumps Orchitis & Group 1 & $0 / 1(0 \%)$ \\
& Group 2 & $1 / 1(100 \%)$ \\
& Overall & $1 / 4(25 \%)$ \\
Klinefelter Syndrome & Group 1 & $0 / 2(0 \%)$ \\
& Group 2 & $1 / 2(50 \%)$ \\
& Overall & $0 / 4(0 \%)$ \\
& Group 1 & $0 / 2(0 \%)$ \\
& Group 2 & $0 / 2(0 \%)$
\end{tabular}

In Group 1, viable sperm was present in 19/66 for a viable SRR of $28.7 \%$ while in Group 2, 56/82 patients had sperm identified yielding a $68 \%$ SRR $(p<0.0001)$. Cryopreservation was performed in 7/19 (37\%) in Group 1 and 28/56 (50\%) in Group 2 to be used for future ICSI $(\mathrm{p}=0.427)$.

In those men where sperm were identified, we did not find a statistical difference between Group 1 and Group 2 for average sperm concentration, which was 2.7 sperm per HPF (range 1-6 sperm/HPF) and 2.8 sperm per HPF (range $1-6$ sperm / HPF) $(p=0.88)$ respectively. This was profoundly different from those 71 men with obstructive azoospermia undergoing TESA using the same technique who were excluded from the study. The average sperm concentration in men with OA was 4.5 sperm/ HPF (range 1-20 sperm/ HPF) which is highly statistically different when compared to the average overall NOA sperm concentration of 2.7 ( $p$ $=0.002)$. The average OA sperm concentration was also 
found to be statistically different compared to the sperm concentration of Group $1(\mathrm{p}=0.01)$ and Group $2(\mathrm{p}=$ 0.004) alone.

There were no complications in any of the men including prolonged pain, infection, hematoma, hydrocele or testicular atrophy, as reported by patients or upon physical examination during the follow up period.

\section{DISCUSSION}

Comparing TESA with micro-TESE yields a difficult debate as no randomized study has compared the rates of short- and long-term consequences for these surgical approaches. A review of the literature shows the micro-TESE SRR to be between 38-63\% in NOA patients ${ }^{[6-9]}$. Given the SRR results from this study particularly in men with normal range $\mathrm{FSH} \leq 10 \mathrm{IU} / \mathrm{L}$, we found that TESA in our experience had similar results compared to micro-TESE. TESA does have its merits and should be considered in men with NOA for several reasons.

The first advantage of TESA is the less invasive nature of the procedure. TESA offers an approach that spares the testis and eliminates the need for an incision. On the other hand, micro-TESE requires an open testicular approach, which may decrease the volume of seminiferous tubules within the testicular parenchyma $^{[10]}$. Open biopsies have also been shown to have a higher incidence of intra-testicular bleeding and hematoma compared with percutaneous biopsy ${ }^{[11]}$.

TESA should also be considered due to its simplicity and the cost- effectiveness of the procedure compared with micro-TESE particularly for the individuals with NOA and a normal FSH. Micro-TESE is not generally performed in general urologic practice due to the special surgical skills, which requires a substantial learning curve as well as the need for an operating microscope and general anesthesia. TESA can be performed under local anesthesia with or without intravenous sedation (thereby reducing the risks and cost associated with general anesthesia). In fact, a majority of patients have reported no pain or only mild discomfort during TESA when spermatic cord block alone was used ${ }^{[12]}$.

TESA also requires less operating time than the more technically challenging micro-TESE ${ }^{[7]}$. Most cases of micro-TESA can take hours to obtain spermatozoa. Dabaja et al reported that the mean micro-TESE operative time was 1.8 hours (range 0.5-6.6 hours) for successful micro-TESE and 2.7 hours (range 0.6-7.5 hours) for unsuccessful micro-TESE ${ }^{[8]}$. In our experience, the average operating time for TESA is 0.4 hours (range $0.2-0.8$ hours).
Another advantage to the TESA procedure is a consistently low complication rate. There is only one reported complication requiring surgical correction, which was testicular bleeding managed with hemostatic suturing ${ }^{[2,3,13-17]}$. Hematomas can occur and tend to reabsorb within 1-4 weeks ${ }^{[8]}$. In our cohort, there were no complications. Comaratively, no acute clinical complications after micro-TESE have been published ${ }^{[18,19]}$. However, scrotal ultrasound evaluations done after micro-TESE show evidence of testicular changes including persistent hematoma and inflammation found up to three months after micro$\mathrm{TESE}^{[18-20]}$. In addition to structural changes, microTESE can result in functional changes to the testicle as demonstrated by the report by Ramasamy et $a l^{[21]}$. Their study found that patients who underwent microTESE, had a decline in average testosterone by $20 \%$ $(p<0.01)^{[21]}$.

Preoperative serum FSH level of azoospermic men has long been investigated as a prognostic indicator to correlate with TESA outcomes. Dajani found a strong positive correlation between serum FSH and the presence of mature sperm obtained via TESA when FSH was $<10 \mathrm{IU} / \mathrm{l}^{[22]}$. Mourad et al. found a discernable difference in the average FSH of those who underwent unsuccessful verses successful sperm retrieval from testicular aspiration at $19 \mathrm{IU} / \mathrm{L}$ and 10 IU/L respectively ${ }^{[23]}$. In the report by Nowroozi, the average serum FSH level was lower in those with successful sperm retrieval at $13 \mathrm{IU} / \mathrm{L}$ compared to $23.2 \mathrm{IU} / \mathrm{L}$ in those without mature sperm retrieval ${ }^{[3]}$. In Nowroozi's study, men with serum FSH $<15$ IU/1 and a histology indicating hypo-spermatogenesis had a comparable sperm retrieval rate for TESA vs. TESE ${ }^{[3]}$. Although it is difficult to ascertain the optimal level of FSH for successful SRR given the variation in reported values, all of these aforementioned studies indicate that an elevated FSH portend a lower probability of obtaining viable spermatozoa with TESA. Our study finds a stark difference in SRR in those NOA patients with $(\leq 10 \mathrm{IU} / \mathrm{L})$ vs. those NOA with $>10 \mathrm{IU} / \mathrm{L})$. It is important to note that men in Group 1 should not be discouraged from undergoing sperm retrieval as our study shows $30 \%$ chance of successful sperm retrieval with TESA. It may be that this group would be better served with more extensive exploration using micro-TESE. Although we define the "normal" FSH as $<10 \mathrm{IU} / \mathrm{L}$ based on our laboratory's reference range, we realize that this notion is difficult to generalize as the normal FSH reference range can vary between institutions. The value of this cutoff needs to be further analyzed in future studies using receiver operating characteristic (ROC) analysis with a larger cohort.

Our study does have several limitations. One clear weakness of this study is the absence of diagnostic testicular biopsies and genetic testing such as $\mathrm{Y}$ 
chromosome analysis prior to TESA. Another limitation to this study is not having ICSI success and pregnancy rates. We will address each of these weaknesses.

Diagnostic biopsies have not been obtained as part of our sperm retrieval protocol since 1999. A clear benefit of micro-TESE is having diagnostic biopsies which would allow a more accurate assessment of NOA. However, it is important to note that some argue against performing diagnostic testicular biopsy as it may not be a fair predictor of successful sperm retrieval ${ }^{[24]}$. Diagnostic biopsy can miss focal areas of sperm production that can otherwise be recovered by TESA, which surveys the testicles diffusely and there is the added time and cost of histological assessment. In addition, pre-operative biopsy may cause inflammatory changes, hematoma, or scarring of the testes, which can further deteriorate the quality of any existing spermatogenesis ${ }^{[24]}$. Despite not having confirmatory biopsy, we found that the average sperm concentration of those successfully retrieved in Group 2 to be highly statistically different from our OA group ( $\mathrm{p}$ value 0.004), supporting that the individuals in Group 2 did not have an obstructive process in which sperm concentration is expected to be much higher.

We reconciled the absence of diagnostic biopsies by subcategorizing our population into NOA with an elevated FSH, as can be seen in Sertoli Cell Only syndrome and early maturation arrest, and NOA with normal FSH which is most often associated with late maturation arrest as suggested by Lipshultz ${ }^{[25]}$. He found that men with late maturation arrest, in which spermatids are present, had decreased FSH and a higher TESE sperm retrieval rate compared to those with early maturation arrest, in which primarily spermatogonia or spermatocytes were $\operatorname{seen}^{[25]}$.

Routine genetic testing has also not been performed at our institution. The rationale for doing this test is that having Y- microdeletion or karyotype data may yield information which would obviate TESA if AZFa or $\mathrm{AZFb}$ microdeletions were found and may reclassify these patients to absolute NOA. Yet, in spite of the AUA Best Practice and ASRM Practice Guidelines, which state that genetic testing in men with NOA is important, we have found that out of pocket expense for these tests may exceed the TESA costs ${ }^{[26,27]}$. Given the very low published prevalence of $\mathrm{AZFa}$ or $\mathrm{AZFb}$ microdeletions in azoospermic men of $10-15 \%$, we elected not to pursue this test and with the patients consent moved directly to TESA ${ }^{[28,29]}$.

In the absence of diagnostic biopsies and genetic work-up in our cohort, the genital physical examination is critical to make the diagnosis of presumed NOA. In our experience, we found that the testicular size of less than $4.6 \mathrm{~cm}$ with the absence of clinically obstructive findings is consistent with the non-obstructive process of the disease as confirmed by a relative low number of sperm retrieval as compared to those who have obstructive azoospermia.

Finally, a clear limitation to our study is the absence of ICSI success and live birth rate data. The finding of spermatozoa represents the most significant step prior to fertility treatment on the male side

\section{CONCLUSION}

In summary, our report demonstrates TESA to be an efficient method for obtaining sperm for ICSI or cryopreservation with particular high success of SRR in non-obstructive azoospermic men with normal range FSH. TESA is a valuable surgical method as it is a quicker, easier, less invasive and likely a less costly approach than micro-TESE. In cases of negative retrieval rates after TESA, micro-TESA can still be pursued for sperm retrieval. Future studies should evaluate larger populations of men with NOA to determine the diagnostic value of FSH levels in SRR using ROC analysis as well as assess outcomes for fertilization and live birth rates.

\section{CONFLICT OF INTEREST}

There are no conflicts of interest.

\section{REFERENCES}

1. Willott, G. M. Frequency of Azoospermia Forensic Sci Int. 1982; 20 (1): 9-10.

2. Hauser, R., Yogev, L., Paz, G., Yavetz, H., Azem, F. et al. Comparison of Efficacy of Two Techniques for Testicular Sperm Retrieval in Nonobstructive Azoospermia: Multifocal Testicular Sperm Extraction Versus Multifocal Testicular Sperm Aspiration. J of Androl. 2006; 27(1): 28-33.

3. Nowroozi, M. R., Ahmadi, H., Ayati, M., Jamshidian, H., \& Sirous, A. Testicular FineNeedle Aspiration Versus Testicular Open Biopsy: Comparable Sperm Retrieval Rate in Selected Patients. Indian J of Urol. 2012; 28(1): 37-42.

4. Beliveau M, Turek P. The Value of Testicular Mapping in Men With Non-Obstructive Azoospermia. Asian J Androl. 2011; 13(2):22530 . 
5. Schoor RA, Elhanbly S, Niederberger CS. The Role of Testicular Biopsy in the Modern Management of Male Infertility. J Urol. 2001; 167:197-200.

6. Ishikawa, T. Surgical Recovery of Sperm in Nonobstructive Azoospermia. Asian J Androl 2012; 14(1): 109-115.

7. Ramasamy, R., Reifsnyder, J. E., Husseini, J., Eid, P. A., Bryson, C. et al. Localization of Sperm During Microdissection Testicular Sperm Extraction in Men with Non-obstructive Azoospermia. J Urol. 2013; 189(2): 643-646.

8. Dabaja, A. A., \& Schlegel, P. N. Micro-dissection Testicular Sperm Extraction: An Update. Asian J Androl. 2013; 15(1): 35-39.

9. Maduriera C, Cunha M, Sousa M, Neto A P, Pino $\mathrm{M} J$ et al. Treatment by Testicular Sperm Extraction and Intracytoplastmic Sperm Injection of 65 Azoospermic Patients with Non- Mosaic Klinefelter Syndrome with Birth of 17 Healthy Children. Androl. 2014; 4: 623-631.

10. Tash, J. A., \& Schlegel, P. N. Histologic Effects of Testicular Sperm Extraction on the Testicle in Men with Non-obstructive Azoospermia. Urol. 2001; 57(2): 334-337.

11. Harrington, T. G., Schauer, D., \& Gilbert, B. R. Percutaneous Testis Biopsy: An Alternative to Open Testicular Biopsy in the Evaluation of the Subfertile Man. J Urol. 1996; 156(5): 1647-1651.

12. Gorgy A, Podsiadly BT, Bates S, Craft IL. Testicular Sperm Aspiration: The Appropriate Technique. Hum Reprod. 1998; 13(4): 1111-1113.

13. Donoso, P., Tournaye, H., \& Devroey, P. Which is the Best Sperm Retrieval Technique for Nonobstructive Azoospermia? A Systematic Review. Hum Reprod Update. 2007; 13(6): 539-549.

14. Lisek, E. W., \& Levine, L. A. Percutaneous Technique for Aspiration of Sperm From the Epididymis and Testicle. Techniques in Urol. 1997; 3(2): 81-85.
15. Rosenlund, B., Kvist, U., Ploen, L., Rozell, B. L., Sjoblom, P. et al. Comparison Between Open and Percutaneous Needle Biopsies in Men with Azoospermia. Hum Reprod. 1998; 13(5): 12661271.

16. Khadra, A. A., Abdulhadi, I., Ghunain, S., \& Kilani, Z. Efficiency of Percutaneous Testicular Sperm Aspiration as a Mode of Sperm Collection for Intracytoplasmic Sperm Injection in Nonobstructive Azoospermia. J Urol. 2003; 169(2): 603-605.

17. Lewin A, Reubinoff B, Porta-Katz A, Weiss $\mathrm{D}$, Eisenberg $\mathrm{V}$ et al. Testicular Fine Needle Aspiration: The Alternative Method for Sperm Retrieval in Non-Obstructive Azoospermia. Hum Reprod. 1999; 14(7): 1785-90.

18. Amer M, Ateyah A, Hany R, Zohdy W. Prospective Comparative Study Between Microsurgical and Conventional Testicular Sperm Extraction in NonObstructive Azoospermia: Follow up by Serial Ultrasound Examinations. Hum Reprod. 2000; 15: 653-656.

19. Schlegel PN. Testicular Sperm Extraction: Microdissection Improves Sperm Yield with Minimal Tissue Excision. Hum Reprod. 1999; 14:131- 135 .

20. Okada H, Dobashi M, Yamazaki T, Hara I, Fujisawa $\mathrm{M}$, et al. Conventional Versus Microdissection Testicular Sperm Extraction for Nonobstructive Azoospermia. J Urol. 2002;168:1063-7.

21. Ramasamy, R., Yagan, N., \& Schlegel, P. N. Structural and Functional Changes to the Testis After Conventional Versus Microdissection Testicular Sperm Extraction. Urol. 2005; 65(6): 1190-1194.

22. Dajani YF, Kilani Z. Role of Testicular Fine Needle Aspiration in The Diagnosis of Azoospermia. Int $\mathbf{J}$ Androl 1998; 21:295-300.

23. Mourad WA, Tulbah A, Merdad T, Shoukri M, Al Dayel $F$ et al. Fine-Needle Aspiration of the Testis in Azoospermic Men: The Value of Measuring Serum Follicle Stimulating Hormone 
and Testicular Size. Diagn Cytopathol. 2005; 32: $185-8$.

24. Ramasamy R, Lin K, Veeck G, Rosenwak Z, Palermo $G$ et. al. High Serum FSH Levels in Men with Nonobstructive Azoospermia Does Not Affect Success of Microdissection Testicular Sperm Extraction. Fertil Steril. 2009; 92: 590593.

25. Weeden J, Bennett R, Fenig D, Lamb D, Lipshultz L. Early Versus Late Maturation Arrest: Reporductive Outcomes of Testicular Failure. J Urol. 2011; 186(2):621-6.

26. Jarow J, Sigman M, Kolettis P, Lipshultz $\mathrm{L}$, McClure $\mathrm{R}$ et al. The Evaluation of the
Azoospermic Male: American Urol Assoc Best Pract Statement. 2011.

27. American Society for Reproductive Medicine.: Evaluation of the Azoospermic Male Fertil Steril. 2008; 90: 74-77.

28. Foresta C, Ferlin A, Moro E et al. Y Chromosome Microdeletions and Alterations of Spermatogenesis. Endocr Rev. 2001; 22(2):22639.

29. Ma, K., Mallidis, C. and Bhasin, S. The Role of Y Chromosome Deletions in Male Infertility. Eur. J. Endocrinol. 2000; 142: 418-430. 